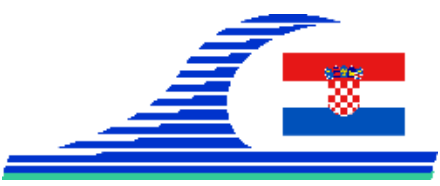

\title{
Statistical analysis and probabilistic modeling of chloride ingress spatial variability in concrete coastal infrastructures
}

\author{
Franck SCHOEFS ${ }^{1}$, Mestapha OUMOUNI ${ }^{1}$, Romain CLERC ${ }^{1}$, \\ Inès OTHMEN ${ }^{2}$, Stéphanie BONNET ${ }^{2}$
}

1. Université de Nantes, UFR Sciences et Techniques, GeM, UMR 6112 CNRS, IUML, FR CNRS 34 73, 2 rue de la Houssinière, BP 92208, 44322 Nantes, France.

2. Université de Nantes, IUT de Saint-Nazaire, 58 rue Michel Ange 44600 SaintNazaire, France

\begin{abstract}
:
Chloride ingress is the main deterioration process of reinforced concrete (RC) structures exposed to marine environment. This paper presents experimental studies combined with statistical analysis to assess spatial variability of the main parameters influencing chloride-induced corrosion for $\mathrm{RC}$ i.e. the $\mathrm{RC}$ resistivity $\rho$, the surface chloride content, $C_{s}$, and the chloride diffusion coefficient $D_{a}$. In the maintenance context, they must be evaluated as spatial random fields. Thus, we investigate 36 chloride profiles and 6 resistivity paths measured on the same concrete. The measurement error is incorporated in the model, then statistical estimations and test are performed to assess properties of the random field and the error on the scale of fluctuations.
\end{abstract}

\section{Keywords:}

Chloride ingress, Reinforced concrete, Marine environment, Resistivity, Spatial variability, Random field, Statistical analysis

\section{Introduction}

Chloride ingress is among the main causes of degradation of reinforced concrete (RC) structures where the deterioration is more perceptible in marine environment. Therefore, in view to state maintenance and repair decisions, one must assess model parameters such as the surface concentration $C_{s}$ and the diffusion coefficient $D_{a}$, or physical concrete properties such as concrete resistivity $\rho$ (O'CONNOR \& KENSHEL, 2013). However, those parameters, as others model inputs or concrete properties, show considerable spatial variability which is due to the intrinsic randomness of the material composition, environmental conditions (temperature and humidity) and the workmanship quality. Several researches have shown that spatial variability cannot be simplified to a random variable. For instance, recent studies have highlighted the impact of spatial variability on the level of structural reliability (STEWART \& MULLARD, 2007). Thus, parameters $C_{s}, D_{a}$ and $\rho$ are modeled by spatial random fields (SRF). 
Mediterranean rocky coasts:

Features, processes, evolution and problems

However, nowadays, the major challenge of those models is to determine stochastic properties of the SRF, namely the marginal distribution, stationarity and uncertainty on the spatial correlation parameter estimation.

In this work, a 28 years old beam built with the same concrete and located in a marine environment is investigated to model $C_{s}, D_{a}$ and $\rho$ with SRF. Maximum Likelihood Estimate (MLE) combined with statistical tests analysis is performed to assess properties of random fields and the uncertainties on the correlation parameters.

\section{Presentation of the studied beam}

Data sets come from the research operation SVC2 (Spatial Variability of Chloride in Concrete), (DESBOIS et al., 2012). Studied chloride levels and resistivity have been measured on interior and exterior faces of a $R C$ beam of the Montoir-de-Bretagne (France) coal terminal, which has been built on the edge of the Loire, in 1981-1982. Positioned on gateway 5, row 51, the beam, shown on figure 1 , is $0.4 \mathrm{~m}$ wide, $9.34 \mathrm{~m}$ long and $0.86 \mathrm{~m}$ high and situated at $+0.84 \mathrm{~m}$ GLF (General Leveling of France).
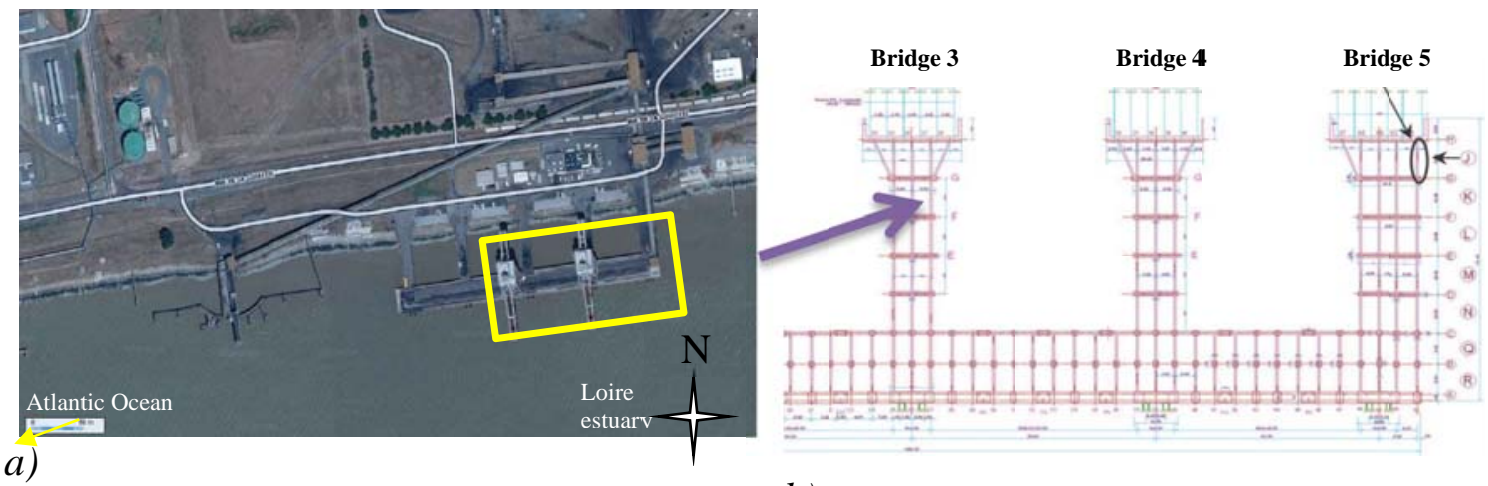

b)
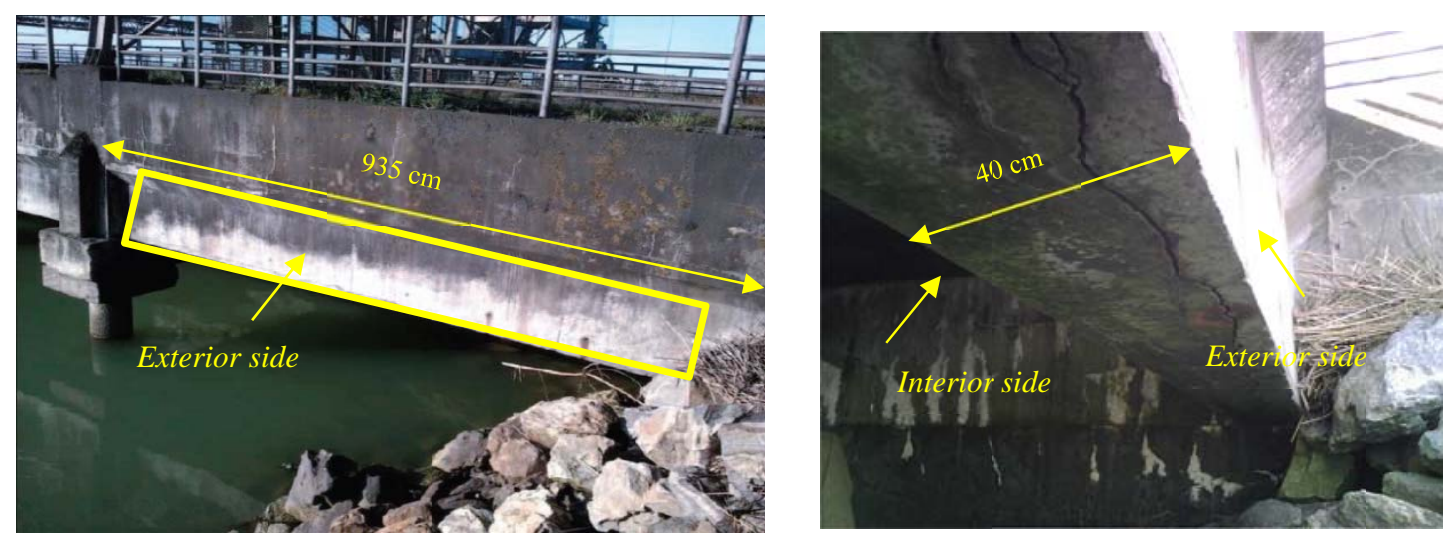

c)

d)

Figure 1. Different views: a) Aerial view, b) Sketch of bridge 3, 4 and 5, c) Beam J, d) Exposed and sheltered side of beam $J$. 
Mediterranean rocky coasts:

Features, processes, evolution and problems

\section{Experimental investigations and results}

\subsection{Resistivity measurements}

Resistivity measurements have been performed using the Resi device from IFSTTAR ${ }^{1}$, which uses Wenner-Proceq resistance probe, with electrodes $5 \mathrm{~cm}$ spaced from each other. Moreover, each measure comes from 2 to 8 diagonal-opposite measurements. These latter points allow to get rid of heterogeneity problems due to aggregates.

We aim to assess the correlation parameter of the resistivity measurements. Concrete is assumed horizontally homogeneous. Thus, six one-dimensional resistivity trajectories are studied. Three on each face, at the beam ordinates $29 \mathrm{~cm}, 45 \mathrm{~cm}$, and $73 \mathrm{~cm}$, respectively called low, medium, and high-level.

Firstly, we test the stationarity of the trajectories with Kwiatkowki-Phillips-SchmidtShin (KPSS) and Dickey-Füller (DF) tests (KWIATKOWSKY et al., 1992; PHILLIPS \& PERRON, 1988). Normal and log-normal law are tested with Kolmogorov-Smirnov (KS) test with the skewness and kurtosis estimation. Secondly, we use MaximumLikelihood Estimate (MLE) to assess, the mean, variance and the scale of fluctuation.

Results obtained with a log-normal law are interesting, where KPSS and KS tests cannot reject the stationarity and normality hypotheses, and DF test (PHILLIPS \& PERRON, 1988) rejects the null of an auto-regressive model. In addition, kurtosis and skewness of log-data are close to standard values (respectively 3 and 0 ). Thus, the field $\rho$ is considered stationary and log-normally distributed. Moreover, we notice clear tendencies on the estimated values of the mean $\mu$, the standard-deviation $\sigma$ and the scale of fluctuation $l_{c}$ (figure 2).

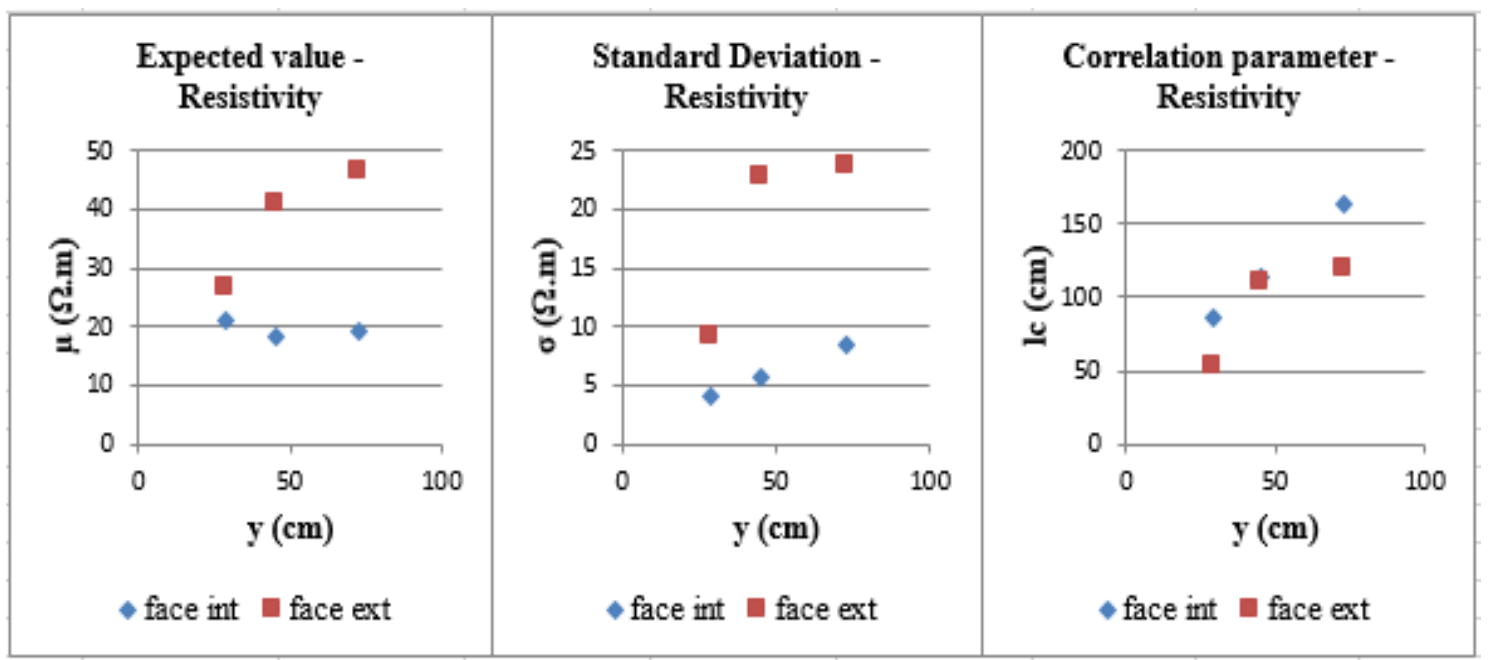

Figure 2. Evolution of $\mu, \sigma$ and $l_{c}$ with the height.

French institute of science and technology for transport, spatial planning, development and networks 
Mediterranean rocky coasts:

Features, processes, evolution and problems

\subsection{Chloride content measurements}

Chloride content measurements were performed on the same beam. The extraction of cylindrical cores was carried out in 2011 after 28 years of exposure. It was realized on a line located at $40 \mathrm{~cm}$ height; 30 samples were collected. They are $30 \mathrm{~cm}$ spaced. The extracted specimens are $5 \mathrm{~cm}$ in diameter and $40 \mathrm{~cm}$ long. Each core was divided into 5 slides; slides 1 and 2 for the exterior side and slides 4 and 5 for interior side. They were used to determine the chloride profiles, using the procedure recommended by the RILEM TC 178-TMC (VENNESLAND et al., 2013). Slide 3 is used for other investigations such as porosity, compressive strength and estimation of the initial chloride content. Fick's second law of diffusion was then fitted to estimate parameters $C_{s}$ and $D_{a}$ from total chloride content $C$.

We check the stationarity for the paths of $C$, statistical test suggests to consider the lognormal distribution for $C, C_{s}$ and $D_{a}$. The inspection is assumed to be imperfect, the measurement errors are modelling by a log-white noise. Then parameters $C, C_{s}$ and $D_{a}$ are describing by the model:

$$
\exp (\sigma G+\tau B)
$$

where the random field $\exp (\sigma G)$ models the hidden parameters and $\tau B$ is a white noise with variance $\tau^{2}$. The spatial random field $G$ is assumed defined by a stationary exponential correlation function $\rho(r)=e^{-r / l_{c}}$. The method MLE is performed to assess the mean, both variances $\sigma^{2}$ and $\tau^{2}$ and the scale of fluctuation of each parameter. Figure 3 shows the behaviour of $\mu$, the total variance $\sigma^{2}+\tau^{2}$ and $l_{c}$ (in $\mathrm{m}$ ) with respect to the depth (in $\mathrm{mm}$ ) for the logarithm of the homogenized paths of chloride profiles. It shows high degree of variability with respect to depth for the interior side than the exterior side. The difference in the mean is due to the environment conditions. The variance can be considered constant in the depth, so the coefficient of the variation is constant almost similar for both sides.

Table 1. Statistics of logarithm of the model, exterior side.

\begin{tabular}{|c|c|c|c|c|c|c|}
\hline ext. side & $\mu$ & $\sigma^{2}$ & $\tau^{2}$ & $l_{c}$ & $l_{c}$ (min) & $I_{c}$ (max) \\
\hline $\log -C_{s}$ & -5.31 & 0.02 & 0.06 & 127 & 0 & 615 \\
\hline $\log -D_{a}$ & -27.37 & 0.18 & 0.014 & 31.17 & 0 & 73 \\
\hline
\end{tabular}

Table 2. Statistics of logarithm of the model, interior side.

\begin{tabular}{|c|c|c|c|c|c|c|}
\hline int. side & $\mu$ & $\sigma^{2}$ & $\tau^{2}$ & $l_{c}$ & $l_{c}$ (min) & $I_{c}$ (max) \\
\hline Log- $C_{s}$ & -4.97 & 0.09 & 0.001 & 19.1 & 0 & 44.32 \\
\hline Log- $D_{a}$ & -27.3 & 0.25 & 0.0027 & 13.1 & 0 & 39.5 \\
\hline
\end{tabular}


Table1 and table 2 give estimate of the mean $\mu$, variances $\sigma^{2}$ and $\tau^{2}$ and the correlation length $l_{c}$ in $(\mathrm{cm})$ and their upper bounds with 95\% asymptotic confidence level. The value of $l_{c}$ of $\log -C_{s}$ is different from the side exterior and interior, the first result with the upper bound can be compared well with previously reported in the literature (ENGELUND \& SORENSEN, 1998; O'CONNOR \& KENSHEL, 2013). However, for the interior side it means a strong variability because high levels of humidity which keeps more chloride. The value of $l_{c}$ of $\log -D_{a}$ is closely linked with the microstructure of the component; this explains the difference between values for both sides. This estimated value was reported by (ENGELUND \& SORENSEN, 1998; O'CONNOR \& KENSHEL, 2013), where it was not based on the analysis of the field data.
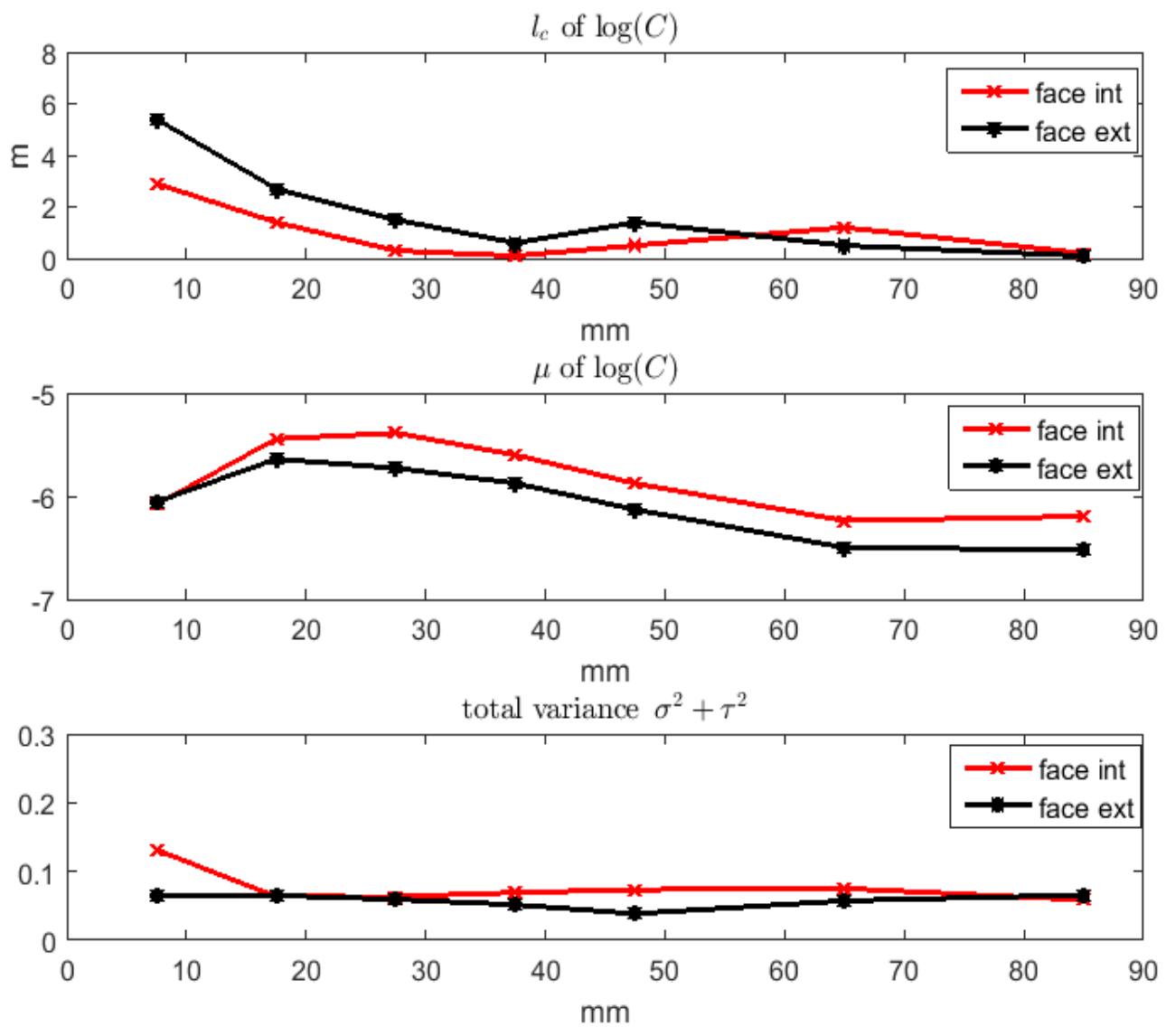

Figure 3. Evolution of $\mu$, $\sigma$ and $l_{c}$ with the depth, (face i.e. side).

\section{Conclusion and discussion}

Firstly, while resistivity mean and standard deviation seem constant on the interior face, they increase with the height on the exterior face. We suppose this is due to different environmental conditions. Secondly, the resistivity scale of fluctuation increases with 
Mediterranean rocky coasts:

Features, processes, evolution and problems

the height in both, which might show the anisotropy and heterogeneity of the material, also different level of degradation between the sides.

On the other hand, these results are compatible with those of chloride content. They show the effect of the humidity on the chlorides ingress. This latter can be modeled with a stationary random field by analyzing results of $\log -D_{a}$ in both sides. Moreover, asymptotic confidence region of the scale fluctuation suggests more measurements to improve estimation. We note that scale fluctuation of both sides have approximately the same behaviour in the deep beam, since the environmental conditions are almost the same. It is also interesting for more reliable study to analyse the uncertainty of the Fick model and the correlation between $\mathrm{D}_{\mathrm{a}}$ and $\mathrm{C}_{\mathrm{s}}$.

Finally, the results obtained in this study are very significance for appropriate modeling of spatial variability in planning maintenance techniques for structures exposed to the marine environment.

\section{References}

DESBOIS T., PASQUIET S., QUEGUINER R., THAUVIN B. (2012). Poste charbonnier, Grand Port Maritime de Nantes-Saint-Nazaire, Montoir de Bretagne Etape 1 - Synthèse des mesures réalisées sur site et avant réparation de l'ouvrage par protection cathodique. Technical report, CETE de l'Ouest, France, 66 p.

ENGELUND S., SORENSEN J. D. (1998). A probabilistic model for chloride Ingress and initiation of corrosion in reinforced concrete structures. Struct. Safety, 20, pp. 6989. doi.org/10.1016/S0167-4730(97)00022-2

KWIATKOWSKY D., PHILLIPS P. C. B., SCHMIDT P., SHIN Y. (1992). Testing the null hypothesis of stationarity against the alternative of a unit root: How sure are we that economic time series have a unit root? Journal of econometrics, Vol. 54 (1-3), pp. 159-178. doi.org/10.1016/0304-4076(92)90104-Y

O'CONNOR A. J., KENSHEL O. (2013). Experimental evaluation of the scale of fluctuation for spatial variability modeling chloride-induced reinforced concrete corrosion. J. of Bridge Eng., V. 18(1), pp.3-14. doi.org/10.1061/(ASCE)BE.1943-5592.0000370 PHILLIPS P. C. B., PERRON P. (1988). Testing for a unit root in time series regression. Biometrika, Vol. 75 (2), pp. 335-346. doi: 10.2307/2336182

STEWART M. G., MULLARD J. A. (2007). Spatial time-dependent reliability analysis of corrosion damage and the timing of first repair for RC structures. Eng. Structures, Vol. 29 (7), pp.1457-1464. doi.org/10.1016/j.engstruct.2006.09.004

VENNESLAND Ø., CLIMENT M. A., ANDRADE C. (2013). Recommendation of RILEM TC 178-TM. Testing and modelling chloride penetration in concrete. Methods for obtaining dust samples by means of grinding concrete in order to determine the chloride concentration profile. Materials and Structures Vol., 46 (3), pp. 337-344.

doi: 10.1617/s11527-012-9968-1 